\title{
Déficit de vitamina B-12 en adultos mayores: ¿Un problema de salud pública en Chile?
}

\author{
HUGO SÁNCHEZ ${ }^{1}$, CECILIA ALBALA ${ }^{1}$, EVA HERTRAMPF ${ }^{1}$, \\ RENATO VERDUGO ${ }^{2}$, MANUEL LAVADOS ${ }^{2}$, JOSE LUIS CASTILLO ${ }^{2}$, \\ LYDIA LERA $^{\text {1a }}$, RICARDO UAUY ${ }^{1}$
}

'Unidad de Salud Pública y Nutrición. Instituto Nutrición y Tecnología en Alimentos, Universidad de Chile. Santiago de Chile.

${ }^{2}$ Departamento de Ciencias Neurológicas, Facultad de Medicina, Universidad de Chile. aEstadística, Doctora en Ciencias Matemáticas.

Trabajo financiado por los proyectos FONDECYT No1070592 у 1080589

Recibido el 9 de julio de 2009, aceptado el 14 de diciembre de 2009.

Correspondencia a: Hugo Sánchez R. INTA, Avda. El Líbano 5524, Casilla 138-11, Santiago, Chile. E-mail: hsanchez@inta.cl

\section{Prevalence of vitamin B-12 deficiency in older adults}

Background: There is a correlation between aging and the decrease of plasma levels of vitamin B-12. Aim: To determine the prevalence of vitamin B-12 and folate deficiency and its hematological impact among older adults (AM). Material and Methods: Cross-sectional study, in 1028 subjects aged 65 to 87 years, living in community and evaluated between 2005 and 2008. Percentile distribution of vitamin B-12, folate, hemoglobin, packed red cell volume and mean cell volume by gender and age were analyzed. Deficiency was defined as vitamin B-12 levels $<148$ pmol/L, marginal deficiency as vitamin B-12 levels $<221$ pmol/L, anemia was defined as an hemoglobin $<13$ and $12 \mathrm{~g} / \mathrm{dL}$ among men and women, respectively. Results: The prevalence of vitamin B-12 deficiency was $12 \%$ and the figure for marginal deficiency was $25.4 \%$. Males were more affected than females $(p<0.001)$. The frequency of anemia was $8.6 \%$, and was higher among women $(p=0.004)$. Conclusions: There is a high prevalence of full blown and marginal deficit of vitamin B-12 among the elderly. This deficiency should be considered for correction through public nutrition policies.

(Rev Med Chile 2010; 138: 44-52).

Key words: Aged; Anemia, macrocytic; Vitamin $B_{12}$.
L as características del envejecimiento de la población en América Latina se originan en los grandes cambios demográficos, epidemiológicos y nutricionales experimentados por la población en las últimas décadas ${ }^{1}$. La velocidad de envejecimiento en la región, más del doble que lo observado en Norteamérica y Europa occiden$\mathrm{tal}^{2}$, ha traído como consecuencia un acelerado aumento de problemas de salud asociados a la vejez, evidenciando la necesidad de identificar los factores de riesgo modificables para enfermedad y discapacidad en este grupo de edad.

El progresivo deterioro biológico relacionado con el envejecimiento es la resultante de la interac- ción de factores genéticos y ambientales; estos últimos incluyen estilos de vida, hábitos alimentarios, actividad física y presencia de enfermedades. En este contexto, la nutrición modula los cambios que provoca el envejecimiento en diferentes órganos y funciones del organismo ${ }^{3}$.

El rol de los micronutrientes es fundamental en los procesos metabólicos de los seres vivos, algunos de ellos con características de esenciales, como es el caso de la vitamina B-12 y los folatos ${ }^{4}$. La vitamina B-12 es sintetizada por bacterias que no son funcionales en seres humanos. Los animales ingieren estos microorganismos incorporándola en sus tejidos, huevos y leche, los que al ser inge- 
ridos por los seres humanos, la incorporan a su organismo.

La vitamina B-12 se almacena en el hígado y las reservas pueden alcanzar hasta aproximadamente $2.500 \mu \mathrm{g}$. Puesto que los requerimientos en seres humanos son bajos ( $2,4 \mu \mathrm{g} /$ día $)$, las manifestaciones clínicas o subclínicas del déficit se presentan bastante tiempo después de un aporte insuficiente (2 a 3 años).

La vitamina B-12 y el acido fólico son micronutrientes primordiales en múltiples vías metabólicas, tanto en la formación de glóbulos rojos, como en el sistema nervioso central (SNC). Ambos están involucrados en procesos de metilación, necesarios para la producción de neurotransmisores, nucleótidos y fosfolípidos ${ }^{5}$.

La alteración de dichos procesos en el SNC puede causar enfermedades neurológicas o psiquiátricas, tales como neuropatía periférica, depresión, edeterioro cognitivo y demencia ${ }^{6-8}$. Es importante destacar que estos síntomas pueden manifestarse en ausencia de alteraciones hematológicas en 20-30\% de los sujetos. Estas cifras pueden ser mayores en presencia de un aumento de la ingesta de ácido fólico, en especial cuando se asocian suplementos a la fortificación con niveles altos de ácido fólico?.

La presentación hematológica más habitual de la carencia de B-12 es la anemia megaloblástica. Considerando que esta anemia es en todo similar a la producida por déficit de folatos, en Chile, a partir del inicio del programa de fortificación de la harina de trigo con ácido fólico en el año $2000^{10}$, es una enfermedad que se diagnostica cada vez con menos frecuencia.

Se ha observado que existe una correlación entre el envejecimiento y el descenso de los niveles plasmáticos de vitamina B-12, al punto de que alrededor de $20 \%$ de los mayores de 60 años presentan esta insuficiencia ${ }^{11}$.

El objetivo del estudio fue determinar la prevalencia de déficit de vitamina B-12 y folatos, y su impacto en la serie roja hematológica de los adultos mayores.

\section{Sujetos y Métodos}

Estudio de tipo transversal, analítico, en una muestra de 1.028 adultos mayores que viven en la comunidad (rango edad: 65 a 87 años), evaluados entre 2005 y 2008 , provenientes de las determina- ciones basales de diferentes estudios de cohorte realizados por nuestro grupo: Estudio $\mathrm{SABE}^{12}$, Estudio CENEX ${ }^{13}$ y Estudio "Impacto funcional de la deficiencia subclínica de vitamina B-12 sobre las esferas cognitiva y de neuroconducción en adultos mayores. Evaluación controlada de un programa de suplementación" FONDECYT No1070592 (ISRCTN 02694183), actualmente en desarrollo. Los sujetos fueron invitados a participar y citados para su evaluación, la cual incluyó una muestra de sangre en ayunas. Los valores séricos de vitamina B-12 y folatos se determinaron mediante radioinmunoensayo (Dual Count Solid phase no boil Assay Siemmens, LA). Las muestras fueron procesadas en duplicado. El análisis de la serie roja incluyó: hemoglobina ( $\mathrm{Hb} ; \mathrm{g} / \mathrm{dL})$, hematocrito (Hto: \%) y volumen corpuscular medio (VCM: fL); estos parámetros se determinaron con un contador electrónico de partículas Cell-Dyn Model 1700 (Abbott Diagnostics, Abbott Park, IL).

Análisis Estadístico: Se efectuó un análisis por grupos de edad, sexo y distribución percentilar de la vitamina B-12, folatos, hemoglobina, hematocrito y volumen corpuscular medio. Para la categorización del estatus de vitamina B-12, se consideraron como déficit los valores menores de $148 \mathrm{pmol} / \mathrm{L}$; como deficiencia marginal, los valores entre $148 \mathrm{pmol} / \mathrm{L}$ y $221 \mathrm{pmol} / \mathrm{L}$; y, como normales, los mayores a $221 \mathrm{pmol} / \mathrm{L}^{14}$. En el caso de los folatos, los valores menores de $7 \mathrm{nmol} / \mathrm{L}$ se consideraron como déficit; como normales, los valores entre 7 y $46 \mathrm{nmol} / \mathrm{L}$; y, elevados, los valores $>46 \mathrm{nmol} / \mathrm{L}^{15}$. Las prevalencias se expresan en porcentaje e IC 95\%. Para su análisis estadístico se utilizó el test $\chi^{2}$ estableciéndose un nivel de confianza del $95 \%$. El diagnóstico de anemia se efectuó con valores de hemoglobina $<13 \mathrm{~g} / \mathrm{dL}$ en hombres $\mathrm{y}<12 \mathrm{~g} / \mathrm{dL}$ en mujeres ${ }^{16}$. Y el de microcitosis y de macrocitosis, con valores $\langle 80 \mathrm{fL} \mathrm{y}>$ $100 \mathrm{fL}$, respectivamente ${ }^{17}$.

Para el análisis de correlación, se utilizó el coeficiente de Pearson, considerando como variables independientes el nivel plasmático de vitamina B-12 y el de folatos y, como variables dependientes, los niveles plasmáticos de hemoglobina, hematocrito y volumen corpuscular medio.

Se realizó una regresión logística para determinar los factores de riesgo de anemia y déficit de vitamina B-12 o de folatos. Como variables independientes, se incluyeron el sexo, la edad y los niveles plasmáticos de vitamina B-12 y de folatos; 
como variables dependientes, la presencia de anemia, de valores de B-12<221 pmol/L, y de valores de folatos $<7 \mathrm{nmol} / \mathrm{L}$.

Se estableció el nivel de confianza en 95\%. Los análisis fueron realizados con STATA 10.

\section{Resultados}

La estructura de la muestra por grupo de edad y sexo se presenta en la Tabla 1, observándose una relación 2:1 de mujeres:hombres, obteniéndose una muestra de sujetos decreciente al aumentar la edad, de forma tal que cambios en relación con la edad posiblemente corresponden a sobrevida diferencial.

Como se observa en la Tabla 2, se observan diferencias significativas en las medianas de vita- mina B-12 y folatos; entre hombres y mujeres y entre grupos de edad. Se observa que los valores considerados como déficit para vitamina B-12 y folatos se encuentran bajo el percentil 10 y se

Tabla 1. Estructura de la muestra por grupo de edad y sexo

\begin{tabular}{|lccc|}
\hline $\begin{array}{l}\text { Grupo de } \\
\text { Edad }\end{array}$ & Hombres & $\begin{array}{c}\text { n (\%) } \\
\text { Mujeres }\end{array}$ & Total \\
$65-69$ años & $149(45,3)$ & $377(53,9)$ & $526(51,2)$ \\
\hline $70-79$ años & $168(51,1)$ & $310(44,3)$ & $478(46,5)$ \\
\hline 80 y más & $12(3,6)$ & $12(1,7)$ & $24(2,3)$ \\
\hline Total & $329(100)$ & $699(100)$ & $1.028(100)$ \\
\hline
\end{tabular}

Tabla 2. Distribución percentilar de vitamina B-12, folatos y hemoglobina

\begin{tabular}{|c|c|c|c|c|c|}
\hline \multirow{2}{*}{$\begin{array}{l}\text { Sexo/edad } \\
\text { Vitamina B-12 (pmol/L) }\end{array}$} & \multicolumn{5}{|c|}{ Percentiles } \\
\hline & $\mathbf{P}_{10}$ & $\mathbf{P}_{25}$ & $\mathbf{P}_{50}$ & $\mathbf{P}_{75}$ & $\mathbf{P}_{90}$ \\
\hline \multicolumn{6}{|l|}{ Hombres* } \\
\hline $65-69 * *$ & 171,2 & 263,4 & 379,5 & 569,7 & 851,6 \\
\hline $70-79 * *$ & 68,5 & 133,9 & 212,2 & 320,1 & 431,0 \\
\hline 80 y más** & 157,9 & 170,8 & 310,0 & 776,0 & 885,6 \\
\hline Total & 102,1 & 167,5 & 289,1 & 447,4 & 694,7 \\
\hline \multicolumn{6}{|l|}{ Mujeres* } \\
\hline $65-69 * *$ & 221,3 & 331,4 & 519,2 & 756,8 & $1.009,3$ \\
\hline $70-79 * *$ & 128,0 & 199,2 & 276,7 & 394,9 & 584,5 \\
\hline 80 y más** & 217,7 & 227,7 & 329,1 & 497,8 & 885,6 \\
\hline Total & 152,0 & 244,0 & 375,4 & 610,1 & 887,8 \\
\hline Folatos (nmol /L) & $\mathbf{P}_{10}$ & $\mathbf{P}_{25}$ & $\mathbf{P}_{50}$ & $\mathbf{P}_{75}$ & $\mathbf{P}_{90}$ \\
\hline \multicolumn{6}{|l|}{ Hombres* } \\
\hline $65-69 * *$ & 28,1 & 31,7 & 38,1 & 43,0 & 47,9 \\
\hline $70-79 * *$ & 12,7 & 22,0 & 33,1 & 40,1 & 47,3 \\
\hline 80 y más** & 19,2 & 20,2 & 31,5 & 35,4 & 45,8 \\
\hline Total & 17,4 & 28,1 & 35,3 & 41,5 & 47,7 \\
\hline \multicolumn{6}{|l|}{ Mujeres* } \\
\hline $65-69 * *$ & 29,3 & 33,5 & 39,8 & 45,0 & 50,2 \\
\hline $70-79 * *$ & 15,0 & 26,0 & 34,7 & 40,8 & 47,3 \\
\hline 80 y más** & 21,1 & 22,3 & 29,1 & 38,4 & 43,0 \\
\hline Total & 22,5 & 31,0 & 37,6 & 43,3 & 49,2 \\
\hline
\end{tabular}

*Diferencia entre sexos $p<0,05$; * Diferencia entre grupos de edad $p<0,05$ 
incrementan con la edad. Destaca que 15,9\% (IC95\%: 13,7-18,3) de los sujetos presentan niveles considerados como suprafisiológicos para folatos (> $46 \mathrm{nmol} / \mathrm{L}$ ), de los cuales $6,1 \%$ presentaron déficit de vitamina B-12. En la Figura 1 se observa la distribución de las concentracion de vitamina B-12 por sexo, observándose valores inferiores en hombres.

En la Tabla 3 se describe la distribución percentilar de Hto, Hb, VCM, encontrándose que los niveles de hemoglobina son significativamente menores en mujeres y el VCM aumenta con la edad, en ambos sexos $(\mathrm{p}<0,05)$. Se observó una correlación positiva significativa $(R=0,12)$ entre edad y VCM. Sin embargo, sólo 0,5\% (n: 5) de sujetos presentó macrocitosis, de ellos sólo 3 tenían valores de déficit de vitamina B-12 y uno de estos, valores bajos de folatos, no presentando ninguno de ellos anemia. No se observó correlación entre la edad con el hematocrito ni la hemoglobina $(\mathrm{p}>0,05)$.

Como se detalla en la Tabla 4, la prevalencia del déficit y déficit marginal de vitamina B-12 (< $221 \mathrm{pmol} / \mathrm{L}$ ) aumentó en los sujetos con mayor edad ( $\mathrm{p}<0,001$ ). Es destacable que $25,4 \%$ de los AM de ambos sexos, presentaron valores de vitamina $\mathrm{B}-12<221 \mathrm{pmol} / \mathrm{L}$. Al analizar por sexo, los hombres presentaban prevalencias significativamente mayores que las mujeres, con valores de 37,1\% (IC95\%: 31,8-41,5) y 19,9\% (IC95\%:
$17,0-23,0)$, respectivamente $(\mathrm{p}<0,001)$; hecho que se mantuvo al analizar por grupos de edad. El déficit de folatos sólo se observó en una mujer de 71 años, la cual además presentaba déficit de vitamina B-12 y anemia.

La prevalencia de anemia fue de 8,6\% (IC95\%: $6,9-10,4)$ en la muestra total. Al desagregar por sexo, se observó que las mujeres presentaron una prevalencia de 10,3\% (IC95\%:8,1-12,8), cifra significativamente mayor que el 4,9\% (IC 95\%: $2,8-7,8)$ observado en los hombres $(\mathrm{p}=0,004)$. En las mujeres el grupo de 70-79 años presentó una prevalencia de anemia de 14,8\% (IC95\%: 11,1-19,3), cifra significativamente mayor que lo encontrado en los otros grupo de edad ( $\mathrm{p}=$ 0,001). El 5,7\% de los sujetos con anemia presentó microcitosis y ninguno presentó macrocitosis. $\mathrm{Al}$ analizar la relación entre déficit de vitamina B-12 y presencia de anemia, no se observó diferencias significativas en el análisis general de la muestra $(\mathrm{p}=0,295)$, ni por sexo, ni por grupos de edad. Se observó una correlación negativa entre edad/ vitamina B-12 (R: -0,30) y edad/folatos (R: -0,27)

Como se observa en la Tabla 5, los factores de riesgo de presentar déficit de vitamina B-12 son: ser hombre, con un riesgo el doble al de las mujeres y la edad avanzada. En el caso del riesgo de presentar anemia se observó que las mujeres tienen 2,46 veces más riesgo que los hombres y tambien la edad avanzada.

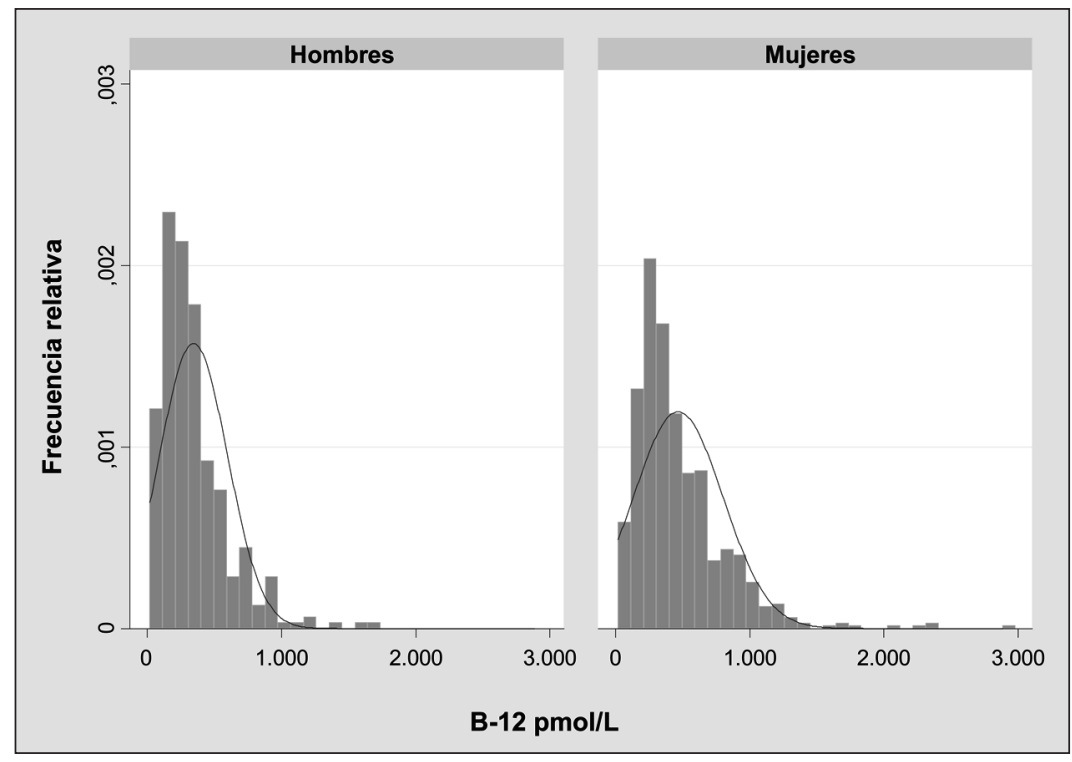

Figura 1. Histograma de valores de concentración de Vitamina B-12 plasmática por sexo. 
Tabla 3. Distribución percentilar de hematocrito, hemoglobina y VCM

\begin{tabular}{|c|c|c|c|c|c|}
\hline \multirow{2}{*}{$\begin{array}{l}\text { Sexo/edad } \\
\text { Hematocrito(\%) }\end{array}$} & \multicolumn{5}{|c|}{ Percentiles } \\
\hline & $\mathbf{P}_{10}$ & $\mathbf{P}_{25}$ & $\mathbf{P}_{50}$ & $\mathbf{P}_{75}$ & $\mathbf{P}_{90}$ \\
\hline \multicolumn{6}{|l|}{ Hombres* } \\
\hline $65-69$ & 39,8 & 41,7 & 43,7 & 45,7 & 47,5 \\
\hline $70-79$ & 39,4 & 41,3 & 43,4 & 45,3 & 47,5 \\
\hline 80 y más & 37,6 & 39,7 & 41,8 & 46,0 & 48,2 \\
\hline Total & 39,5 & 41,3 & 43,5 & 45,4 & 47,5 \\
\hline \multicolumn{6}{|l|}{ Mujeres* } \\
\hline $65-69$ & 35,9 & 37,8 & 39,4 & 41,1 & 42,6 \\
\hline $70-79$ & 35,2 & 37,6 & 39,7 & 41,4 & 43,4 \\
\hline 80 y más & 38,3 & 39,2 & 40,2 & 41,4 & 43,0 \\
\hline Total & 35,6 & 37,7 & 39,5 & 41,3 & 42,9 \\
\hline Hemoglobina (g/dL) & $\mathbf{P}_{10}$ & $\mathbf{P}_{25}$ & $\mathbf{P}_{50}$ & $\mathbf{P}_{75}$ & $\mathbf{P}_{90}$ \\
\hline \multicolumn{6}{|l|}{ Hombres* } \\
\hline $65-69$ & 13,4 & 14,1 & 14,9 & 15,5 & 16,3 \\
\hline $70-79$ & 13,4 & 14,0 & 14,8 & 15,4 & 16,3 \\
\hline 80 y más & 12,7 & 13,7 & 14,3 & 15,5 & 16,2 \\
\hline Total & 13,4 & 14 & 14,9 & 15,5 & 16,3 \\
\hline \multicolumn{6}{|l|}{ Mujeres* } \\
\hline $65-69$ & 12,2 & 12,7 & 13,3 & 13,9 & 14,4 \\
\hline $70-79$ & 11,8 & 12,5 & 13,3 & 14,0 & 14,5 \\
\hline 80 y más & 13,1 & 13,2 & 13,8 & 14,1 & 14,8 \\
\hline Total & 11,9 & 12,6 & 13,3 & 13,9 & 14,5 \\
\hline VCM(fL) & $\mathbf{P}_{10}$ & $\mathbf{P}_{25}$ & $\mathbf{P}_{50}$ & $\mathbf{P}_{75}$ & $\mathbf{P}_{90}$ \\
\hline \multicolumn{6}{|l|}{ Hombres* } \\
\hline $65-69 * *$ & 85 & 87 & 89 & 92 & 96 \\
\hline $70-79 * *$ & 86 & 88 & 91 & 93 & 96 \\
\hline 80 y más** & 84 & 86,5 & 89,5 & 92 & 93 \\
\hline Total & 85 & 88 & 90 & 93 & 96 \\
\hline \multicolumn{6}{|l|}{ Mujeres* } \\
\hline $65-69 * *$ & 83 & 86 & 88 & 91 & 93 \\
\hline $70-79 * *$ & 84 & 87 & 90 & 92 & 94 \\
\hline 80 y más** & 88 & 90 & 91 & 92,5 & 94 \\
\hline Total & 83 & 86 & 89 & 92 & 93 \\
\hline
\end{tabular}

*Diferencia entre sexos $p<0,05 ;$ ** Diferencia entre grupos de edad $p<0,05$ 
Tabla 4. Prevalencia de déficit de vitamina B-12 en adultos mayores

\begin{tabular}{|c|c|c|c|c|}
\hline & $\begin{array}{c}\text { Déficit } \\
<148 \mathrm{pmol} / \mathrm{L}\end{array}$ & $\begin{array}{l}\text { Déficit marginal } \\
148-221 \mathrm{pmol} / \mathrm{L}\end{array}$ & $\begin{array}{c}\text { Normal } \\
>221 \mathrm{pmol} / \mathrm{L}\end{array}$ & \\
\hline Hombres* & $\begin{array}{c}N(\%) \\
(\mathrm{Cl} 95 \%)\end{array}$ & $\begin{array}{c}N(\%) \\
(\mathrm{Cl} 95 \%)\end{array}$ & $\begin{array}{c}\text { N (\%) } \\
(\mathrm{Cl} 95 \%)\end{array}$ & Total \\
\hline $65-69 * *$ & $\begin{array}{c}12(8,0) \\
(4,2-13,6)\end{array}$ & $\begin{array}{c}15(10,1) \\
(5,7-16,1)\end{array}$ & $\begin{array}{l}122(81,9) \\
74,7-87,7\end{array}$ & $149(100)$ \\
\hline $70-79 * *$ & $\begin{array}{c}47(28,0) \\
(21,3-35,4)\end{array}$ & $\begin{array}{c}44(26,2) \\
(19,7-33,5)\end{array}$ & $\begin{array}{c}77(45,8) \\
(38,1-53,7)\end{array}$ & $168(100)$ \\
\hline 80 y más** & $\begin{array}{c}1(8,3) \\
(0,2-38,5)\end{array}$ & $\begin{array}{c}3(25,0) \\
(5,5-57,2)\end{array}$ & $\begin{array}{c}866,7) \\
(34,9-90,1)\end{array}$ & $12(100)$ \\
\hline Total & $\begin{array}{c}60(18,2) \\
(14,2-22,8)\end{array}$ & $\begin{array}{c}62(18,8) \\
(14,7-23,5)\end{array}$ & $\begin{array}{l}207(62,9) \\
(57,4-68,1)\end{array}$ & $329(100)$ \\
\hline Mujeres* & $\begin{array}{c}N(\%) \\
(\mathrm{Cl} 95 \%)\end{array}$ & $\begin{array}{c}\mathbf{N}(\%) \\
(\mathrm{Cl} 95 \%)\end{array}$ & $\begin{array}{c}\text { N (\%) } \\
(\mathrm{Cl} 95 \%)\end{array}$ & Total \\
\hline $65-69 * *$ & $\begin{array}{l}20(5,3) \\
(3,2-8,0)\end{array}$ & $\begin{array}{c}17(4,5) \\
(2,6-7,1)\end{array}$ & $\begin{array}{c}340(90,2) \\
(86,7-93,0)\end{array}$ & $377(100)$ \\
\hline $70-79 * *$ & $\begin{array}{c}43(13,9) \\
(10,2-18,2)\end{array}$ & $\begin{array}{c}57(18,4) \\
(14,2-23,1)\end{array}$ & $\begin{array}{l}210(67,7) \\
(62,2-72,9)\end{array}$ & $310(100)$ \\
\hline 80 y más** & $0(0)$ & $\begin{array}{c}2(16,7) \\
(2,1-48,4)\end{array}$ & $\begin{array}{c}10(83,3) \\
(51,6-97,9)\end{array}$ & $12(100)$ \\
\hline Total & $\begin{array}{c}63(9,0) \\
(7,0-11,4)\end{array}$ & $\begin{array}{l}76(10,9) \\
(8,6-13,4)\end{array}$ & $\begin{array}{c}560(80,1) \\
(76,9-83,0)\end{array}$ & $699(100)$ \\
\hline Total & $\begin{array}{c}\text { N (\%) } \\
(\mathrm{Cl} 95 \%)\end{array}$ & $\begin{array}{c}\text { N (\%) } \\
(\mathrm{Cl} 95 \%)\end{array}$ & $\begin{array}{c}\text { N (\%) } \\
(\mathrm{Cl} 95 \%)\end{array}$ & Total \\
\hline $65-69 * *$ & $\begin{array}{l}32(6,1) \\
(4,2-8,5)\end{array}$ & $\begin{array}{l}32(6,1) \\
(4,2-8,5)\end{array}$ & $\begin{array}{c}462(87,8) \\
(84,7-90,5)\end{array}$ & $526(100)$ \\
\hline $70-79 * *$ & $\begin{array}{c}90(18,8) \\
(15,4-22,6)\end{array}$ & $\begin{array}{c}101(21,1) \\
(17,5-25,1)\end{array}$ & $\begin{array}{l}287(60,0) \\
(55,5-64,5)\end{array}$ & 478 (100) \\
\hline 80 y más** & $\begin{array}{c}1(4,2) \\
(0,1-21,2)\end{array}$ & $\begin{array}{c}5(20,8) \\
(7,1-42,1)\end{array}$ & $\begin{array}{c}18(75,0) \\
(53,3-90,2)\end{array}$ & $24(100)$ \\
\hline Total & $\begin{array}{c}123(12,0) \\
(10,0-14,1)\end{array}$ & $\begin{array}{c}138(13,4) \\
(11,4-15,7)\end{array}$ & $\begin{array}{c}767(74,6) \\
(71,8-77,2)\end{array}$ & $1.028(100)$ \\
\hline
\end{tabular}

*Test $\chi^{2}$ : Diferencia entre sexos $p<0,05 ;{ }^{*}$ Test $\chi^{2}$ : Diferencia entre grupos de edad $p<0,05$

Tabla 5 Factores de riesgo para déficit de Vitamina B-12 y anemia

\begin{tabular}{|lcc|}
\hline & $\begin{array}{c}\text { Déficit Vitamina B-12 } \\
(<\mathbf{2 2 1} \mathbf{~ p m o l / L )} \\
\text { OR (IC95\%) }\end{array}$ & Anemia \\
Sexo Femenino & $0,45(0,33-0,60)$ & OR (IC95\%) \\
Edad & $1,11(1,08-1,16)$ & $2,46(1,39-4,35)$ \\
B-12 $(<221 \mathrm{pmol} / \mathrm{L})$ & & $1,06(1,01-1,11)$ \\
Folatos & $0,96(0,95-0,98)$ & $1,38(0,86-2,36)$ \\
\hline
\end{tabular}




\section{Discusión}

El déficit de B-12 (< 148 pmol/L) fue de 12\% en ambos sexos, cifra superior a las comunicadas en estudios extranjeros e inferior a lo encontrado en otros de menor tamaño realizados en Chile. En la última encuesta NHANES 2003-2004, sólo 3,9\% (CI95\%: 2,9-5,2) de la población estadounidense de 60 y más años presentó esta condición ${ }^{18}$. Clarke et $\mathrm{al}^{19}$, en el Reino Unido, han informado que entre $3 \%$ y $28 \%$ de los adultos de 65 y más años presenta cifras menores de $150 \mathrm{pmol} / \mathrm{L}$, dependiendo de la cohorte estudiada y que la prevalencia de déficit de vitamina B-12 aumenta en sujetos de mayor edad. En Chile, Olivares et al informaron que, en mayores de 60 años, 50,5\% de los hombres y $33,1 \%$ de las mujeres presentan déficit de vitamina B-12 ${ }^{16}$. Hirsch et al, en tanto, encontraron, después de dos años de iniciado el programa de fortificación de la harina de trigo con ácido fólico, una prevalencia de déficit de vitamina B-12 de $27,6 \%$ en sujetos de 70 y más años ${ }^{20}$. Los resultados dispares entre los estudios mencionados y el nuestro pueden atribuirse a la estructura etaria de las poblaciones estudiadas, dada la relación directa entre el aumento en la edad y la mayor prevalencia de déficit. Cabe destacar que, en nuestra población, los adultos mayores de sexo masculino tienen mayor riesgo de presentar valores inferiores a los normales $(\mathrm{B}-12<221 \mathrm{pmol} / \mathrm{L})$.

Por otro lado, es necesario señalar como limitación de nuestro estudio el escaso número de adultos de 80 y más años, lo cual podría explicar los cambios en las prevalencias de déficit de vitamina B-12 encontradas.

Luego de iniciada en Chile la fortificación con ácido fólico $(2,2 \mathrm{mg} / \mathrm{kg}$ de harina), los niveles plasmáticos de folatos en ancianos se incrementaron casi al doble, como lo demuestra el estudio de Hirsch et al. Más aún, nuestros datos demuestran que en este grupo la deficiencia de folatos ya no existe. Por otra parte, $15 \%$ de nuestros sujetos presenta valores considerados suprafisiológicos, aunque éstos no alcanzan la magnitud de los encontrados en los grupos de ancianos norteamericanos, donde al aporte de ácido fólico de la fortificación, se agrega el de los suplementos ${ }^{10}$.

Es destacable que la anemia encontrada no se relaciona con el déficit de folatos, ni de vitamina B-12 (ausencia de macrocitosis), ni de hierro (microcitosis sólo en 1,6\% de los sujetos), lo cual sugiere un posible origen inflamatorio, como ha sido descrito tanto por Olivares et al, en ancianos chilenos, como por otros autores a nivel internacional $^{21}$. Otra posible causa de anemia que podría ser estudiada, son las enfermedades de la médula ósea, particularmente el grupo de los síndromes mielodisplásicos, los cuales provocan cierto grado de macrocitosis. Los factores de riesgo encontrados en nuestro estudio para presentar anemia fueron el sexo femenino y la edad avanzada.

Las altas prevalencias encontradas de déficit y déficit marginal de vitamina B-12 en la población adulta mayor que vive en comunidad nos plantea distintos desafíos.

En primer lugar, reconocer esta realidad como un problema de salud pública en la población adulta mayor, más aún dada su relación con problemas cognitivos o de la neuroconducción ${ }^{22}$. Observaciones no publicadas por nuestro grupo han demostrado la alta frecuencia de estas últimas alteraciones en la población de 70 y más años, no diabética, asociada a déficit de B-12 (FONDECYT 1070592).

En segundo lugar, determinar las causas que generan este déficit en la poblacion adulta mayor. Estudios internacionales mencionan como posibles causas: a) la atrofia gástrica asociada al proceso de envejecimiento que dificulta la liberación de la vitamina B-12 de los alimentos ${ }^{23,24}$, que conlleva a la disminución del factor intrínseco, elemento clave en la absorción de la vitamina B-12 en el intestino ${ }^{25,26} \mathrm{y}$ b) la disminución de la ingesta de productos animales que contienen B-12. Datos preliminares de nuestro estudio en curso sugieren que la ingesta promedio de los adultos mayores con déficit de vitamina B-12 está por sobre las recomendaciones diarias de ingesta (INR) para este grupo de edad, por lo cual es necesario profundizar en el estudio del primer factor mencionado.

Por último, definir cuál es la mejor conducta frente a valores de vitamina B-12 bajo el límite aceptado en la actualidad ( $148 \mathrm{pmol} / \mathrm{L})$, tanto en el contexto individual como en el poblacional. En el caso individual se requiere una evaluación clínica que permita establecer la existencia de alteraciones funcionales en el ámbito hematológico o neurológico, el diagnóstico causal de la deficiencia, tratamiento y control permanente del paciente.

En modelos de regresión, en los cuales se relaciona ingesta y niveles plasmáticos de vitamina B-12, ha sido posible estimar que fortificaciones/ 
suplementaciones $(\mathrm{F} / \mathrm{S})$ con vitamina B-12 > 500 $\mu \mathrm{g} /$ día, llevarían los niveles plasmáticos a cifras superiores de $221 \mathrm{pmol} / \mathrm{L}$. Se ha observado que la F/S con vitamina B-12 cristalina, predice mejor los niveles plasmáticos de este micronutriente; esta forma química se absorbe de igual manera en individuos con y sin atrofia de la mucosa gástrica ${ }^{23}$. En Chile, el programa de alimentación complementaria para el adulto mayor de 70 y más años, entrega un aporte de 58,3\% de los requerimientos diarios de vitamina B-12. Estudios sugieren que una vez que la deficiencia se ha establecido en sujetos con malabsorción, terapias con 40 a $80 \mu \mathrm{g} /$ día de vitamina B-12 cristalina oral por 30 días no revierte los signos de deficiencia ${ }^{27,28}$.

En nuestra población se agrega un riesgo más, dado que los adultos mayores chilenos tienen asegurado su aporte de ácido fólico, es posible encontrar la combinación de niveles plasmáticos bajos de vitamina B-12 y niveles suprafisiológicos de folatos séricos, la cual generaría un mayor riesgo para el estado neurocognitivo de los adultos mayores. Esta combinación aparece en 1,0\% de los sujetos estudiados. Estudios recientes sugieren que niveles suprafisiológicos de folato sérico con déficit de vitamina B-12 aumentan el deterioro $\operatorname{cognitivo}^{22,29}$. Hay consenso internacional sobre la necesidad de asegurar un adecuado aporte de vitamina B-12, en especial a los adultos mayores, en caso de existir una fortificación universal con ácido fólico ${ }^{27,30}$.

En el contexto poblacional, serán necesarias intervenciones para mejorar el estado de vitamina B-12, manteniendo una estrecha vigilancia sobre interacciones adversas con ácido fólico. Para ello será necesario definir la mejor alternativa de prevención primaria del déficit de vitamina B-12 en esta población, siendo esta, a través de suplementación medicamentosa o de un alimento fortificado con vitamina B-12. Esta decisión debiera tomarse a la luz de la mejor información disponible, colocando en la balanza los riesgos y beneficios de una posible intervención para mejorar los niveles plasmáticos de vitamina B-12 en la población adulta mayor.

\section{Referencias}

1. Albala C, Vio F, Kain J, Uauy R. Nutrition Transition in Latin America: The Case of Chile. Nutr Reviews 2001;
56: 170-6.

2. Palloni A, Hill K, Pinto Aguirre G. Economic swings and demographic changes in the history of Latin America. Popul Stud (Camb) 1996; 50: 105-32.

3. Berry E. Chronic Disease: How can Nutrition Moderate the Effects? Nutrition Reviews 1994; 52: S28-S30.

4. Shils M, Shike M, Ross C, Caballero B, Cousins R. Eds. Modern Nutrition in Health and Disease. Tenth Edition. Lippincott, Williams and Wilkins. Baltimore, MD, 2006.

5. Carmel R, Green R, Rosenblat DS, Watkins D. Update on cobalamin, folate and homocysteine, Hematology 2003; 62-81.

6. Penninx B, Guralnik J, Ferrucci L, Fried L, Allen R, Stabler S. Vitamin B-12 Deficiency and Depression in Physically Disabled Older Women: Epidemiologic Evidence From the Women's Health and Aging Study. Am J Psychiatry 2000; 157: 715-21.

7. Nogales J, Jiménez P, García P, Sáez D, Aracena R, González J, et al. Mielopatía por déficit de vitamina B-12: caracterización clínica de 11 casos. Rev Med Chile 2004; 132: 1377-82.

8. Behrens MI, Díaz V, Vásquez C, Donoso A. Demencia por déficit de vitamina B-12. Caso clínico. Rev Med Chile 2003; 131: 915-9.

9. Lindenbaum J, Healton EB, Savage DG, Brust JC, Garrett TJ, Podell ER, et al. Neuropsychiatric disorders caused by cobalamin deficiency in the absence of anaemia or macrocytosis. N Engl J Med 1988; 318: 1720-8.

10. Hertrampf E, Cortés F, Erickson D, Cayazzo M, Freire W, Bailey LB, et al. Consumption of folic acid-fortified bread improves folate status in women of reproductive age in Chile. J Nutr 2003; 133: 3166-9.

11. Allen LH. How common is vitamin B-12 deficiency? Am J Clin Nutr 2009; 89: 693S-6S.

12. Albala C, Lebrão ML, León Díaz EM, Ham-Chande R, Hennis AJ, Palloni A, et al. Encuesta Salud, Bienestar y Envejecimiento (SABE): Metodología de la Encuesta y Perfil de la Población Estudiada. Rev Panam Salud Pública 2005; 7: 307-22.

13. Dangour AD, Albala C, Aedo C, Elbourne D, Grundy E, Walker D, Uauy R. A factorial-design cluster randomised controlled trial investigating the cost-effectiveness of a nutrition supplement and an exercise programme on pneumonia incidence, walking capacity and body mass index in older people living in Santiago, Chile: The CENEX study protocol. Nutr J 2007; 6: 14.

14. Allen L. Folate and vitamin B-12 Status in the Americas. Nutrition Reviews 2004; 62: S29-S33.

15. Clarke R, Evans G, Schneede J, Nexo E, Bates C, Fletcher A, et al. vitamin B-12 and folate deficiency in later life. Age and Ageing 2004; 33: 34-41. 
16. Olivares M, Hertrampf E, Capurro MT, Wegner D. Prevalence of anemia in elderly subjects living at home: role of micronutrient deficiency and inflammation. Eur J Clin Nutr 2000; 54: 834-9.

17. WHO/UNICEF/UNU, eds. Iron deficiency anaemia assessment prevention, and control: a guide for programme managers. Geneva, Switzerland: World Health Organization, 2001.

18. Pfeiffer CM, Johnson CL, Jain RB, Yetley EA, Picciano MF, Rader JI, et al . Trends in blood folate and vitamin B-12 concentrations in the United States, 1988-2004. Am J Clin Nutr 2007; 86: 718-27.

19. Clarke R, Evans G, Schneede J, Nexo E, Bates C, Fletcher A, et al. vitamin B-12 and folate deficiency in later life. Age and Ageing 2004; 33: 34-41.

20. Hirsch S, De la Maza P, Barrera G, Gattas V, Petermann M, Bunout D. The Chilean Flour Folic Acid Fortification program reduces serum homocysteine levels and masks B-12 deficiency in elderly people. J Nutr 2002; 132: 28991.

21. Andrès E, Federici L, Serraj K, Kaltenbach G. Update of nutrient-deficiency anemia in elderly patients. Eur J Intern Med. 2008; 19: 488-93.

22. Morris MS, Jacques PF, Rosenberg IH, Selhub J. Folate and vitamin B-12 status in relation to anemia, macrocytosis, and cognitive impairment in older Americans in the age of folic acid fortification. Am J Clin Nutr 2007; 85: 193-200.
23. Campbell A, Millar J, Green R, Hann M, Allen L. Plasma Vitamin B-12 concentrations in an Elderly Latino population are predicted by serum Gastrin concentrations and crystalline vitamin B-12 intake. J Nutr 2003; 133: 2770-6.

24. Russell RM. Changes in gastrointestinal function attributed to aging. Am J Clin Nutr 1992; 55: 1203S-7S

25. Miller A, Furlong D, Burrows BA, Slingerland W, Bound vitamin B-12 absorption in patients with low serum B-12 levels. Am J Hematol 1992; 40: 163-6.

26. Scarlett JD, Read H, O'Dea K. Protein-bound cobalamin absorption declines in the elderly. Am J Hematol 1992; 39: 79-83.

27. Cuskelly GJ, Mooney KM, Young IS Folate and vitamin B-12: friendly or enemy nutrients for the elderly. Proc Nutr Soc 2007; 66: 548-58.

28. Selhub J, Morris MS, Jacques PF. In vitamin B-12 deficiency, higher serum folate is associated with increased total homocysteine and methylmalonic acid concentrations. Proc Natl Acad Sci USA 2007 (11); 104 (50): 19995-20000.

29. Selhub J, Morris MS, Jacques PF, Rosenberg IH. Folatevitamin B-12 interactionin relation to cognitive impairment, anemia, and biochemical indicators of vitamin B-12 deficiency. Am J Clin Nutr 2009; 89: 702S-6S.

30. Johnson MA. If High Folic Acid Aggravates Vitamin B-12 Deficiency What Should Be Done About It?. Nut Rev 2007; 65: 451-8. 